\title{
Endocrine
}

\section{Comparison of different systems of (US) risk stratification for malignancy in elderly patients with thyroid nodules. Real world experience.

\author{
--Manuscript Draft--
}

Manuscript Number:

Full Title:

Article Type:

Corresponding Author:

Corresponding Author Secondary

Information:

Corresponding Author's Institution:

Corresponding Author's Secondary Institution:

First Author:

First Author Secondary Information:

Order of Authors:

Funding Information:

\section{Order of Authors Secondary Information:}

\section{Abstract:}

ENDO-D-19-01110R3

Comparison of different systems of (US) risk stratification for malignancy in elderly patients with thyroid nodules. Real world experience.

\section{Original Article}

Gabriela Brenta

Dr. Cesar Milstein Care Unit

ARGENTINA

Dr. Cesar Milstein Care Unit
Fernando Di Fermo

Fernando Di Fermo

Noelia Sforza

Melanie Rosmarin

Morosan Allo

Carina Parisi

Jimena Santamaria

Nestor Pacenza

Carlos Zuk

Cristina Faingold

Florencia Ferraro

Tomas Meroño

Gabriela Brenta

Purpose: To comparatively assess the performance of three sonographic classification systems, American Thyroid Association(ATA), the American College of Radiology Thyroid Imaging Reporting and Data System(ACR TI-RADS), and American Association of Clinical Endocrinologists(AACE)/American College of Endocrinology(ACE)/Associazione Medici Endocrinologi(AME) in identifying malignant nodules in an elderly population.

Methods: Cross-sectional study of patients referred for fine needle aspiration biopsy in an academic center for the elderly. One nodule/patient was considered. Nodules classified BethesdaV/VI were considered malignant. Receiver operating characteristics (ROC) curves were established and compared to evaluate diagnostic performance. Malignancy among biopsies below the size cut-off for each ultrasound classification was also compared.

Results: 1867 patients (92\%females); median(Q1-Q3) age 71(67-76) years, were studied showing $82.8 \%$ benign (Bethesda II) and $2.6 \%$ malignant cytology. The 3 classifications correctly identified malignancy $(P<0.01)$. Nonetheless, in the ATA and 
AACE/ACE/AME 16 and 2 malignant nodules, respectively, were unclassifiable. Including unclassified malignant nodules ( $n=1234$, malignant=50), comparison of the ROC curves showed lower performance of ATA [area under the curve (AUC)=ATA (0.49) vs ACR-TI-RADS (0.62), $p=0.008$ and ATA vs AACE/ACE/AME (0.59), $p=0.022]$. Proportion of below size cut-off biopsies for ATA, ACR-TI-RADS and AACE/ACE/AME was different $[16 \%, 42 \%$ and $29 \%$ (all $p<0.001$ )], but no differences in malignancy rate were observed in these nodules.

Conclusion: The present study is the first to validate in elderly patients these classifications showing that AACE/ACE/AME and ACR TI-RADS can predict thyroid malignancy more accurately than the ATA when unclassifiable malignant nodules are considered. Moreover, in this aged segment of the population, the use of ACR TIRADS avoided more invasive procedures.

\section{Response to Reviewers:}

We would like to thank the Reviewer for all of his/her constructive comments. We have tried to modify the manuscript accordingly with hope that it is now suitable for publication in Endocrine.

We have included a reponse below each comment.

Best regards

Gabriela Brenta

\section{COMMENTS FOR THE AUTHOR:}

Reviewer \#1: The authors addressed all my previous comments. I have no further remarks about the contents.

However, I would suggest double-checking the style of the manuscript, which is very hard to read and follow.

Abbreviations should be used more sparingly. According to the Instructions for the Authors, abbreviations should be defined at first mention and used consistently thereafter.

R. Thank you for these suggestions. We edited the manuscript following these instructions.

It is valid for the Abstract, too: in the current version, the authors use five abbreviations in the Abstract without providing any definition (ATA, ACR, AACE/ACE/AME, US, AUC M). It is true for TIRADS, also.

Furthermore, there is no reason to use (BII) instead of Bethesda II.

Please bear in mind that readers of Endocrine may not be familiar with thyroidologists' jargon.

$R$. Thank you for this suggestion. We edited the abstract anf the rest of the manuscript following these instructions.

In the main manuscript, I suggest avoiding the use of $\mathrm{M}$ (instead of malignant) and $\mathrm{B}$ instead of Bethesda. Also, the use of S for Sensitivity and SP for specificity makes the text harder to read.

R. Thank you for this suggestion. We edited the manuscript following these instructions.

At line 201, PPV is not defined (even if apparently obvious).

On the other hand, the title of Supplementary Table 1 is redundant, including twice the words Sensitivity and Specificity (Sensitivity: Sensitivity (S), Specificity (SP):specificity) $\mathrm{R}$. Thank you for this suggestion. We have defined PPV as positive predictive value and also corrected the title of supplementary table 1.

The use of spacing, semicolons, and "=" should be consistent throughout the text. For example, in line 170 there are both "n=" and "n:".

R. Thank you for this suggestion. We have unified this concept all along the manuscript.

What is the meaning of - NCBI at line 139 ? The reference provided has no connection with the National Center for Biotechnology Information.

R. Thank you for this suggestion. We have removed NCBI that stands for National Center for Biotechnology Information. 
Figure 1 has broken lines and asymmetric boxes.

R. Thank you for this suggestion. We changed the figure following the Reviewer's instructions .

I would recommend careful and meticulous editing of the whole text.

$\mathrm{R}$. Thank you for this suggestion. We edited the manuscript following these instructions. 


\section{Comparison of different systems of ultrasound (US) risk stratification for malignancy in elderly patients} with thyroid nodules. Real world experience.

Di Fermo, Fernando ${ }^{1}$; Sforza, Noelia ${ }^{1}$; Rosmarin, Melanie ${ }^{1}$; Morosan Allo, Yanina ${ }^{1}$; Parisi, Carina ${ }^{1}$;

Santamaria, Jimena ${ }^{1}$; Pacenza, Nestor ${ }^{1}$; Zuk, Carlos$^{2}$; Faingold, Cristina ${ }^{1}$; Ferraro, Florencia ${ }^{3}$; Meroño, Tomas $^{3}$; Brenta, Gabriela ${ }^{1}$.

1. Endocrinology Department, Cesar Milstein Hospital, Buenos Aires CABA, Argentina.

2. Radiology Department, Cesar Milstein Hospital, CABA, Buenos Aires, Argentina.

3. Clinical Biochemistry, School of Pharmacy and Biochemistry. University of Buenos Aires, CABA, Argentina.

Corresponding Author
Gabriela Brenta, M.D.

Department of Endocrinology and Metabolism

Dr. Cesar Milstein Hospital

951 La Rioja Street

Buenos Aires, Argentina

Tel. 54-11-49591500

Email: gbrenta@gmail.com

Orcid number: 0000000155317652

Key Words: Elderly patients, ultrasound characteristics, thyroid nodules, malignant cytology.

Acknowledgements: To the technicians and fellows of the Radiology Department who assisted in filling out the ultrasound characteristics forms.

We thank Dr. Pol Castellano Escuder for his support on ROC curve comparison and other statistical analyses. 


\section{ABSTRACT}

2 Purpose: To comparatively assess the performance of three sonographic classification systems, American

3 Thyroid Association(ATA), the American College of Radiology Thyroid Imaging Reporting and Data

4 System(ACR TI-RADS), and American Association of Clinical Endocrinologists(AACE)/American College of

5 Endocrinology(ACE)/Associazione Medici Endocrinologi(AME) in identifying malignant nodules in an elderly

6 population.

7 Methods: Cross-sectional study of patients referred for fine needle aspiration biopsy in an academic center for

8 the elderly. One nodule/patient was considered. Nodules classified BethesdaV/VI were considered malignant.

9 Receiver operating characteristics (ROC) curves were established and compared to evaluate diagnostic performance. Malignancy among biopsies below the size cut-off for each ultrasound classification was also 11 compared.

12 Results: 1867 patients (92\%females); median(Q1-Q3) age 71(67-76) years, were studied showing 82.8\% 13 benign (Bethesda II) and $2.6 \%$ malignant cytology. The 3 classifications correctly identified malignancy 14 (P<0.01). Nonetheless, in the ATA and AACE/ACE/AME 16 and 2 malignant nodules, respectively, were 15 unclassifiable. Including unclassified malignant nodules $(\mathrm{n}=1234$, malignant $=50)$, comparison of the ROC 16 curves showed lower performance of ATA [area under the curve (AUC)=ATA (0.49) vs ACR-TI-RADS (0.62), $17 p=0.008$ and ATA vs AACE/ACE/AME (0.59), $p=0.022]$. Proportion of below size cut-off biopsies for ATA, 18 ACR-TI-RADS and AACE/ACE/AME was different [16\%, 42\% and 29\% (all $\mathrm{p}<0.001$ )], but no differences in 19 malignancy rate were observed in these nodules.

20 Conclusion: The present study is the first to validate in elderly patients these classifications showing that 21 AACE/ACE/AME and ACR TI-RADS can predict thyroid malignancy more accurately than the ATA when 22 unclassifiable malignant nodules are considered. Moreover, in this aged segment of the population, the use of 23 ACR TI-RADS avoided more invasive procedures. 


\section{INTRODUCTION}

The prevalence of thyroid nodular disease may vary if studied by palpation of the thyroid gland

31 (detected nodules in about $5 \%$ of the population) or by ultrasound (US) (65\% or more individuals with a nodule

32 in the thyroid gland) [1].

Age is a risk factor for nodular disease since thyroid nodules are more frequently found in the elderly

34 than in the general population [2]. In fact, it has been shown that multinodular thyroid disease is $30 \%$ more

35 common in individuals over 70 years of age [3]. These data are in line with the presence of larger goiters

36 associated with a retro sternal presentation and deviation of the trachea at advanced age [4]. Interestingly, the

37 risk of malignancy in thyroid nodules may decrease in elderly patients [3]. It has been previously described that

38 between ages 20 to 60 years, with each passing year, there is a $2.2 \%$ reduction in the relative risk of a nodule

39 to become malignant. In fact, at 20-29 years, each nodule harbors $14.8 \%$ risk of malignancy while that

40 proportion drops to $5.6 \%$ after the age of 70 [3]. However, in the elderly, histological variants are more

41 aggressive [5]. Thus the discovery of thyroid nodules with medullar, anaplastic, poorly-differentiated carcinoma

42 and distant metastasis is more frequent than in the young. Indeed, in patients over 40 years of age there is $7.0 \%$

43 increase per year in the relative risk of finding more aggressive cancer variants, a phenomenon partially

44 explained by a delay in diagnosis [3].

Faced with this scenario it may be speculated that the indiscriminate use of thyroid US can result in 46 overdiagnosis of thyroid nodules in the elderly patients, most of which result benign once biopsied.

47 Nevertheless, a too stringent policy may neglect the early diagnosis of aggressive thyroid cancer present at this 48 age. There are several US classifications that group echographic features of thyroid nodules into categories that 49 stratify its malignant potential and may help to guide fine needle aspiration biopsy (FNAB). Nowadays, 3 50 internationally endorsed sonographic classification systems have been issued, the American Thyroid 51 Association (ATA) [6], the American Association of Clinical Endocrinologists (AACE), American College of 52 Endocrinology (ACE) and Associazione Medici Endocrinologi (AME) [7] and the American College of 53 Radiology Thyroid Imaging Reporting and Data System (ACR TI-RADS) [8],. 
54 Although the elderly population may benefit from the use of any of these 3 US classifications, there is no 55 available data comparing them. The aim of this study is to compare the efficacy of these 3 US classifications in 56 finding malignant cytology in an elderly cohort with thyroid nodules.

\section{METHODS}

\section{Study Cohort and Protocol}

This is a cross-sectional study of consecutive patients with thyroid nodules referred for US guided-

61 FNAB in an academic referral center for the elderly, Dr. Cesar Milstein Hospital, which receives all the patients belonging to the National Institute of Social Services for Retirees and Pensioners (INSSJP) in the city of Buenos Aires, an iodine sufficient metropolitan area. The US features to be analyzed were collected in a consecutive way and their distribution into US guidelines categories was done retrospectively.

Since our hospital is a referral center for FNAB from other medical institutions, biopsy was performed according to the indication of each referring physician. In case of multiple nodules, the presence of suspicious US characteristics was used for the selection of the nodule to be biopsied. Clinical criteria to refer a patient to US guided-FNAB were either a neck mass that was visible or palpable, or that had been found incidentally in 69 a previous imaging study. All US-guided FNABs were carried out by one of 3 operators of our institution, each having more than 20 years experience with this procedure. In order to avoid a large inter-observer variability

71 as previously described for single suspicious features [9], only these 3 experienced clinicians were responsible 72 of describing all the individual US characteristics of the nodules. All this information was filled in by a 73 technician into a specific form online immediately before the FNAB. At the Endocrine Department, this form 74 was used to complete a database with all the information of each patient. Since 2018, the risk categories 75 according to each of the 3 US systems was calculated for each patient and registered in the database.

77 we selected for statistical analysis the one with a malignant cytological finding. If all nodules were benign, the 78 one with the highest US category risk was selected. The decision of one nodule/patient was based on the idea 79 that if several nodules were biopsied but only 1 was malignant; this patient would be referred to surgery based 80 on this specific nodule. Furthermore, in the case of multiple nodules, solid nodules with suspicious US findings 
were the ones initially biopsied considering the other nodules as less relevant. Since this was the criteria chosen

82 by physicians performing the FNAB, the inclusion of the second nodule would render the malignant cytology

83 prevalence to a minimum. This common criteria shared by the sonographists is part of the study of only one-

84 nodule per patient. Last, there were also nodules that were followed in time and were subjected to more than 85 one FNAB during the study period. Given that the same nodule was analyzed at different time points, including 86 every FNAB in the study would have introduced bias. All nodules with Bethesda V/VI cytology were 87 considered malignant. consent form.

71 (67-76) years) were consecutively included for the study. The total amount of biopsied nodules was 2400 but only one nodule per patient was considered. After exclusion of indeterminate (20\% rate of malignancy at our Institution) and insufficient cytology results ( $\mathrm{n}=271)$, a subpopulation of 1596 nodules with benign and malignant cytological results was obtained (Figure 1). Clinical and biochemical characteristics, such as age, sex, previous exposure to radiotherapy, family history of thyroid cancer, personal history of diabetes, thyroid peroxidase antibody (TPOab) positivity and TSH levels, as well as US details were prospectively collected..

The study was approved by the Ethical Committee of our Institution and all patients signed an informed

\section{Image Analysis}

Prior to each biopsy all US characteristics were assessed with real-time US in each thyroid nodule. These included type of echostructure [solid, mixed (>25\% cystic proportion), spongiform and purely cystic], echogenic pattern [Hypoechoic $=$ the nodule echogenicity was compared to normal thyroid (mild hypoechoic) and strap muscles (markedly hypoechoic), isoechoic, hyperechoic, anechoic], margins (irregular or regular), presence of halo (yes or no), microcalcifications (defined as tiny, punctate hyperechoic foci, without comettail sign and distinct of indeterminate hyperechoic spots), macrocalcifications (defined as coarse areas of calcification greater than $1 \mathrm{~mm}$ in size) and the 3 diameters of the nodule in $\mathrm{mm}$. (taller than wide nodules were defined when the anterior-posterior dimension exceeded the axial dimension).

The 3 US classifications: ATA [6], the AACE/ACE/AME [7] and the ACR TI-RADS [8] were applied based on US findings. According to the suspicion of malignancy, the classification proposed by the ATA 
divided nodules into 5 classes: 1 (benign), 2 (very low suspicion), 3 (low suspicion), 4 (intermediate suspicion) and 5 (high suspicion). According to the score ACR TI-RADS, following a sum of points awarded according to the ultrasound findings, nodules were divided into the following levels of suspicion of malignancy: TR 1 (benign), TR 2 (not suspect), TR 3 (very low suspicion), TR 4 (moderately suspect) and TR 5 (highly suspicious) and according to the AACE/ACE/AME guide the risk of malignancy of the lesions was divided into 1 (low risk), 2 (medium) and 3 (high).

FNABs are usually recommended by each US classification above a certain threshold of size. ATA recommends diagnostic FNAB for thyroid nodules $\geq 1 \mathrm{~cm}$ of high suspicion and intermediate suspicion, low suspicion $\geq 1.5 \mathrm{~cm}$ and very low suspicion $\geq 2 \mathrm{~cm}[6]$. The AACE/ACE/AME proposes diagnostic FNAB for thyroid nodules with Class 1 if $\geq 2.0 \mathrm{~cm}+$ increasing size or high-risk history, Class 2 if $\geq 2 \mathrm{~cm}$ and Class 3 if $\geq 1.0 \mathrm{~cm}$ or $\geq 0.5 \mathrm{~cm}+$ subcapsular or paratracheal lesions, suspicious lymph nodes or extrathyroid spread, positive personal or family history of thyroid cancer, history of head and neck irradiation, coexistent suspicious clinical findings (e.g., dysphonia) [7]. The ACR TI-RADS system recommends diagnostic FNAB for thyroid nodules with TR $3 \geq 2.5 \mathrm{~cm}$, TR $4 \geq 1.5 \mathrm{~cm}$, and TR $5 \geq 1 \mathrm{~cm}$ disregarding diagnostic FNAB for TR1 and TR2 122 nodules [8]. In order to investigate the value of these recommendations, all the nodules that were below the 123 recommended size cut-off of each classification were analyzed.

\section{US-guided fine needle aspiration procedure}

A Mindray DC-3 (Shenzhen, China) Doppler-echo machine and a 7.5-10 MHz linear-array probe were used to guide all FNABs in real time. Biopsies were performed using a 23-gauge needle, and visualization of 127 the tip of the needle inside the nodule helped to monitor the correct site for biopsy. At least 2-6 needle passages 128 were performed in each nodule. Material obtained from FNABs was smeared on glass slides, which were 129 immediately placed in 95\% alcohol for Papanicolau stain and sent to the Pathology Department.

\section{Cytological analysis}

This study used the Bethesda System for Reporting Thyroid Cytopathology to describe the cytological 132 results [10]. The results of those nodules included in 2008 were adapted to the Bethesda System. Cytological 133 analysis was performed independently by two pathologists. Validation of this procedure by cyto-histological 
correlation in our Institution was previously reported [11]. Those patients that were referred to surgery at our

135 Institution were also considered for a descriptive analysis $(\mathrm{n}=31)$.

\section{Statistical analysis}

Chi2 and logistic regression were used to evaluate and compare malignant cytology within each US classification. Receiver operating characteristics (ROC) curves were established to compare diagnostic performance. The cut-off with the highest Youden Index was used to calculate the sensitivity and specificity. Since some malignant nodules remained unclassifiable according to the ATA and AACE/ACE/AME US

classification systems, we performed two ROC curve comparison analyses. One after exclusion of

$143(\mathrm{n}=1234,16$ malignant nodules reclassified for ATA and AACE/ACE/AME combined $)$ and the other excluding

144 all unclassifiable nodules whether benign or not $(n=1218$, malignant nodules $=34)$. For paired-comparisons

145 between the area under the curve (AUC) of the US systems we used DeLong method [12].

146 The nodules that were biopsied even when their size was lower than the guidelines recommendations 147 were classified as "below size cut-offs" and pairs were compared using the McNemar test across the different 148 US systems. The false negative (malignant nodules in the "below size cut-offs" biopsies) rate (FNR) was also 149 calculated for each US classification system.

150 Normally distributed variables are presented as mean \pm S.D. and skewed variables as median (Q1-Q3).

151 A $p$ value $<0.05$ was considered significant. Statistical analyses were performed using SPSS 17.0 statistical 152 software (IBM, Chicago, Ill, USA) and R (R Foundation for Statistical Computing, Vienna, Austria) with the 153 'pROC' package [13].

\section{RESULTS}

156 General and US characteristics of the population

157 Out of 1867 patients (92\% females); median (Q1-Q3), age 70 (67-76) years we obtained 82.8\% Benign 158 (Bethesda II) and 2.6\% malignant nodules. Three percent of the patients had previous exposure to radiotherapy, 

$4.2 \%$ family history of thyroid cancer, $14.6 \%$ personal history of diabetes, $31.5 \%$ were TPOab positive and their TSH levels were $1.8(0.9-3.3) \mathrm{mU} / \mathrm{l}$.

With respect to the US characteristics considered suspicious, $75 \%$ were solid nodules ( $\mathrm{n}=1400), 37.5 \%$ mildly hypoechoic ( $n=702), 3.8 \%$ markedly hypoechoic $(n=68), 29 \%$ had a halo $(n=542), 20 \%$ had irregular margins $(n=368)$ and $23 \%$ had a taller than wide shape $(n=428)$. Microcalcifications were found in $13.5 \%$ $(n=252)$ and macrocalcifications in $15 \%(n=285)$. The predominant pattern of central vascularization was found in $15 \%$ of the nodules $(\mathrm{n}=286)$ (Table1).

Out of 50 malignant nodules 31 had surgical confirmation at our institution. Among these tumours 16 (51\%) were classical papillary thyroid cancer and 15 (49\%) other thyroid cancer histotypes= 11 were follicular variants of papillary thyroid cancer, 1 follicular thyroid cancer, 1 medullary thyroid cancer, 2 lymphomas and 1 anaplastic thyroid cancer.

\section{Diagnostic performance of each US-based risk-stratification system}

Malignant cytology within the categories of each US classification was compared. The 3 US classifications correctly identified malignant cytology $(\mathrm{P}<0.01)$ (Table 2). According to the ACR TI-RADS, the proportion of malignant cytology in nodules classified under category TR 3 was $1.9 \%$, under category TR 4, $3.1 \%$ and under category TR 5, $5.8 \%$. Comparing between risk categories, those nodules classified in category TR 5 were at significantly higher risk of being malignant than TR 3 [Odds Ratio $(\mathrm{OR})(95 \% \mathrm{CI})=3.21$ (1.37-7.54), $\mathrm{p}=0.007]$. Nodules classified as ATA low suspicion had a risk of malignancy of $1.3 \%$, under intermediate suspicion of $3.2 \%$ [OR $(95 \% \mathrm{CI})=2.60(1.02-6.62), \mathrm{p}=0.045]$ and under high suspicion of $6.7 \%$ $[\mathrm{OR}(95 \% \mathrm{CI})=5.61(2.47-12.75), \mathrm{p}<0.001 v s$. ATA low suspicion category]. According to AACE/ACE/AME, Class 2 nodules had risk of malignancy of $1.5 \%$ while the risk ascended to $4.7 \%$ in Class 3 [OR $(95 \% \mathrm{CI})=3.23$ $(1.63-6.40), \mathrm{p}<0.001]$.

\section{Comparison of the ATA, ACR TI-RADS, and AACE/ACE/AME, in identifying malignant nodules:}

Since the ATA and AACE/ACE/AME classifications missed 14 and 2 cases of malignant cytology, respectively. After discarding benign unclassifiable nodules, we proceeded to compare the ROC curves of the 3 US systems using two approaches. First, we considered malignant unclassifiable nodules in the lowest risk 
category; and second without any imputation comparing only the nodules that could be classified by the 3 US systems. When malignant unclassifiable nodules were included $(n=1234$, malignant $=50)$, the AUC of ATA was significantly lower than the two other US systems (Table 3). Excluding malignant unclassifiable nodules $(\mathrm{n}=1218$, malignant=34), ROC curve analysis showed the opposite. ATA US classification system had a significantly higher AUC than the others (Table 3 and Supplementary Table 1). Most of the nodules with malignant cytology unclassifiable by ATA were solid but with iso or hyperechogenicity.

When Bethesda III nodules were classified as benign and Bethesda IV as malignant, the results were not different except for an increase in ATA sensitivity and AACE/ACE/AME specificity and an overall increase of positive predictive values (data not shown).

\section{Analysis of FNABs below the recommended size cut-off and unclassifiable nodules}

When we estimated the nodules that were biopsied below size cut-offs we found $16 \%$ for ATA, $42 \%$ for ACR TI-RADS and 29\% for AACE/ACE/AME ( $<<0.001$ for all paired comparisons). Considering the proportion of malignant nodules among these groups as FNR (\%), we obtained similar proportions the 3 US systems [FNR ATA, ACR TI-RADS and AACE/ACE/AME: $1.4 \%$ (3/210), 2.2\% (15/674) and 1.8\% (8/431), respectively, $p=n s]$. On the other hand, both ATA and AACE/ACE/AME left some nodules outside of the classification: $19 \%$ and $5.7 \%$ respectively.

\section{Age tertiles and individual US characteristics}

203 tertile was 71 (70-72) years and in the third tertile was 78 (75-81) years. When analyzed within each age tertile, 204 the proportion of solid echostructure and taller than wide shape was similar between benign and malignant 205 nodules. Hypoechogenicity instead, was significantly more frequent among malignant nodules both in the first $206(p=0.024)$ and second age tertile $(p=0.009)$, but not in the third age tertile $(p=0.224)$. The proportion of irregular 207 margins was significantly higher in malignant nodules along the age tertiles $(p<0.01)$ while microcalcifications 208 were only significantly more frequent in malignant nodules in the third age tertile $(p=0.001)$. With regards to 209 size, it was noted that only in the third age tertile malignant nodules were larger in size than benign ones $210 \quad(32.6 \pm 19.6 \mathrm{~mm}$ vs. $21.9 \pm 9.5 \mathrm{~mm} ; p=0.048)$.

211 


\section{DISCUSSION} used US classifications for malignancy detection, has been tested in an elderly cohort of patients for the first time. All 3 classifications were found to be useful for detection of malignant nodules. Nonetheless, 14 malignant nodules could not be classified by the ATA US system. This shortcoming made the ATA less convenient than the other two US systems. Only in head-to-head comparison using classifiable nodules, the ATA system was slightly superior to ACR TI-RADS and AACE/ACE/AME. Hence, the inability of the ATA classification to identify malignant nodules with iso or hyperechogenicity is of major relevance in aged patients.

Taking into consideration the proposed size cut-off each classification has for recommending FNAB, the ATA showed the lowest proportion of nodules referred to FNAB below the recommended size cut-off level. However, the 3 classifications found similar proportion of malignancy in nodules below the size cut-off level and in general, ACR TI-RADS spared more nodules from being biopsed.

The clinical management of thyroid nodular disease in the elderly represents a challenge. It is known that nodules are more frequent as we age and although most of them are of benign nature, those that are found malignant may pertain to an aggressive variant [3]. Despite the fact that co-morbidities that increase the surgical risk in an older person may discourage the study of nodular disease, the identification of sonographic findings suggesting aggressive malignant disease can help to take a decision [14]. Moreover, US based classifications proposed by different scientific entities are now used to help in malignancy risk stratification. As regards to the ATA and ACR TI-RADS classifications, both retrospective [15] and prospective [16] studies that have compared their diagnostic performances in general population have found similar elevated predictive value of malignancy in high-risk categories.

Most recently, Lauria Pantano et al. [17] also confirmed that the highest risk categories of ATA, AACE/ACE/AME and ACR TI-RADS classifications correctly identified cytologically high-risk thyroid nodules. However, when compared, ACR TI-RADS and AACE/ACE/AME performed better than ATA possibly due to the large amount of non classifiable nodules in the ATA classification. In fact, it was described that non classifiable nodules harbored 7 times higher risk than the "very low suspicion" nodules. In the present study, we also found that the 3 US classifications were reliable to stratify malignancy risk although when only those nodules that could be classified by the 3 systems were compared, the ATA performed better. One possible 
explanation to the discrepancy between the 2 studies may include differences in both populations analyzed. In

241 particular, our study comprised only elderly patients in whom a very low frequency of malignant nodules was 242 detected, in line with previous reports [3] and in contrast to the suggested rate of malignancy proposed by the 243 guidelines for each US classification [6-8]. However, when we decided to include non classifiable nodules in 244 the total population for comparing among US classifications, AACE/ACE/AME and ACR TI-RADS resulted 245 better predictors of malignancy, in agreement with the results of the mentioned study [17].

Furthermore, it was revealed that younger subjects should be considered at higher risk than older ones 247 within the same US category [17]. These findings would also help explain the low specificity of individual 248 suspicious US features found in our study. In fact, it was found that hypoechogenicity, one of the main US 249 characteristics, may lose its diagnostic value as age advances. Similarly, in older women we previously 250 described that for mixed nodules, none of the suspicious US characteristics were associated with malignancy 251 [18]. With regards to microcalcifications in particular, it has been reported that the associated malignant rate 252 differs between younger and older individuals, with a higher yield in patients < 45-years old compared with 253 older patients $[19,20]$. Since the literature in terms of US findings in the elderly is scarce, it could be argued 254 that the higher US risk categories of any classification would be expected to perform less efficiently in older 255 patients.

256 As regards to the efficacy of a US classification to avoid irrelevant biopsies, Grani et al. [21] compared 257 the diagnostic yield of the ATA, AACE/ACE/AME, ACR TI-RADS and 2 other TI-RADS classification 258 systems established by Korean Society of Thyroid Radiology (KSThR) and European Thyroid Association 259 (ETA) and found ACR TI-RADS to have the lowest rate of unnecessary FNAB. Similarly, Xu et al. [22] 260 compared the three newly-updated TI-RADS classification systems by KSThR, ETA and ACR and also found 261 ACR TI-RADS to have the lowest rate of unneeded FNAB. In agreement, in this study it was also found that 262 ACR TI-RADS, due to its higher size threshold to recommend FNAB, yielded a larger proportion of 263 unnecessary biopsies than ATA and AACE/ACE/AME, a finding quite relevant when considering how to avoid 264 invasive procedures in older patients. This potential advantage observed in ACR TI-RADS was also supported 265 by the fact that malignancy rate was similar among nodules below the recommended size threshold in all 3 266 classifications. 
268 offset by the large number of nodules that could not fit in any category such as those that were solid and iso or 269 hyperechoic with at least one suspicious US finding. Moreover, the rate of malignancy in this unclassified group 270 almost reached the malignancy rate in the high risk ATA category. A new approach is therefore needed to unify 271 criteria and create a universal language to report on the US identification of each nodule that can facilitate the 272 implementation of guidelines [23]. Age could ideally be part of this project and larger size cut-offs might be 273 eventually considered according to each patient's age.

274 One of the limitations of this study is the inclusion of only one nodule per patient thus creating a certain 275 bias. However, since benign thyroid nodular disease is more frequent in the elderly, the inclusion of all nodules 276 would have further reduced the low malignancy rate of this cohort.

277 Another questionable finding is the high number of false positive results in the high risk categories. A 278 plausible explanation could be the lack of a unified lexicon deemed necessary to avoid different interpretations 279 of the US features observed during the biopsy. Nevertheless, we relied in the vast experience of 3 high volume 280 operators to define US characteristics which would reduce this bias. An alternate explanation would be that 281 those suspicious US findings reported in the general population are not quite accurate in the elderly. Older 282 patients with long standing multinodular goiters may have nodules of different shapes and exhibit more 283 calcifications that could jeopardize US stratification. In fact, hypoechogenicity was not as specific in the oldest 284 old regarding the younger individuals of this cohort. Considering that only half of the patients with histological 285 confirmation had classical papillary thyroid cancer it could be argued that in the elderly, suspicious US findings 286 that typically are present in this classical variant may not be evident in other forms of thyroid cancer frequent 287 with advanced age. Furthermore, size can be critical since in the subgroup of oldest patients, malignant nodules 288 were larger than benign nodules. It also should be acknowledged as a limitation that all nodules classified as 289 Bethesda V and VI were considered malignant, but not all were submitted to surgery allowing for eventual false 290 positive cases.

291 Strengths of this study were its design with data collected prospectively and consecutively in a single 292 academic center. The number of patients was larger than most studies in the literature and it only included 293 elderly patients which makes it unique. 


\section{CONCLUSION}

The present study is the first to validate in elderly patients 3 US classifications which showed that AACE/ACE/AME and ACR TI-RADS can predict thyroid malignancy more accurately than the ATA classification when all nodules are considered. Moreover, in this aged segment of the population, the use of ACR TI-RADS avoided more invasive procedures.

Also the fact that US suspicious characteristics of thyroid nodules in elderly patients did not result very specific for malignancy might be considered in future guidelines.

\section{COMPLIANCE WITH ETHICAL STANDARDS:}

Funding: This study did not receive funding.

Conflict of Interest: None of the Authors has any conflict of interest.

Ethical approval: All procedures performed in studies involving human participants were in accordance with the ethical standards of the institutional and/or national research committee and with the 1964 Helsinki declaration and its later amendments or comparable ethical standards.

Informed consent: Informed consent was obtained from all individual participants included in the study. 


\section{REFERENCES}

318

319

320

321

322

323

324

325

326

327

328

329

330

331

332

333

334

335

336

337

338

339

340

341

342

343

344

345

346

347

348

349

350

351

352

353

354

355

356

357

358
1. Durante, C., Grani, G., Lamartina, L., Filetti, S., Mandel, S.J., Cooper, D.S.: The Diagnosis and Management of Thyroid Nodules: A Review. JAMA 319(9), 914-924 (2018). doi:10.1001/jama.2018.0898

2. Reiners, C., Wegscheider, K., Schicha, H., Theissen, P., Vaupel, R., Wrbitzky, R., Schumm-Draeger, P.M.: Prevalence of thyroid disorders in the working population of Germany: ultrasonography screening in 96,278 unselected employees. Thyroid 14(11), 926-932 (2004). doi:10.1089/thy.2004.14.926

3. Kwong, N., Medici, M., Angell, T.E., Liu, X., Marqusee, E., Cibas, E.S., Krane, J.F., Barletta, J.A., Kim, M.I., Larsen, P.R., Alexander, E.K.: The Influence of Patient Age on Thyroid Nodule Formation, Multinodularity, and Thyroid Cancer Risk. J Clin Endocrinol Metab 100(12), 44344440 (2015). doi:10.1210/jc.2015-3100

4. Diez, J.J.: Goiter in adult patients aged 55 years and older: etiology and clinical features in 634 patients. J Gerontol A Biol Sci Med Sci 60(7), 920-923 (2005). doi:60/7/920

5. Sorrenti, S., Baldini, E., Tartaglia, F., Catania, A., Arcieri, S., Pironi, D., Calo, P.G., Filippini, A., Ulisse, S.: Nodular thyroid disease in the elderly: novel molecular approaches for the diagnosis of malignancy. Aging Clin Exp Res 29(Suppl 1), 7-13 (2017). doi:10.1007/s40520-016-0654-y

6. Haugen, B.R., Alexander, E.K., Bible, K.C., Doherty, G.M., Mandel, S.J., Nikiforov, Y.E., Pacini, F., Randolph, G.W., Sawka, A.M., Schlumberger, M., Schuff, K.G., Sherman, S.I., Sosa, J.A., Steward, D.L., Tuttle, R.M., Wartofsky, L.: 2015 American Thyroid Association Management Guidelines for Adult Patients with Thyroid Nodules and Differentiated Thyroid Cancer: The American Thyroid Association Guidelines Task Force on Thyroid Nodules and Differentiated Thyroid Cancer. Thyroid 26(1), 1-133 (2016). doi:10.1089/thy.2015.0020

7. Gharib, H., Papini, E., Garber, J.R., Duick, D.S., Harrell, R.M., Hegedus, L., Paschke, R., Valcavi, R., Vitti, P.: American Association of Clinical Endocrinologists, American College of Endocrinology, and Associazione Medici Endocrinologi Medical Guidelines for Clinical Practice for the Diagnosis and Management of Thyroid Nodules--2016 Update. Endocr Pract 22(5), 622-639 (2016). doi:10.4158/EP161208.GL

8. Tessler, F.N., Middleton, W.D., Grant, E.G., Hoang, J.K., Berland, L.L., Teefey, S.A., Cronan, J.J., Beland, M.D., Desser, T.S., Frates, M.C., Hammers, L.W., Hamper, U.M., Langer, J.E., Reading, C.C., Scoutt, L.M., Stavros, A.T.: ACR Thyroid Imaging, Reporting and Data System (TI-RADS): White Paper of the ACR TI-RADS Committee. J Am Coll Radiol 14(5), 587-595 (2017). doi:S1546-1440(17)30186-2

9. Grani, G., Lamartina, L., Cantisani, V., Maranghi, M., Lucia, P., Durante, C.: Interobserver agreement of various thyroid imaging reporting and data systems. Endocr Connect 7(1), 17 (2018). doi:10.1530/EC-17-0336

10. Cibas, E.S., Ali, S.Z.: The Bethesda System for Reporting Thyroid Cytopathology. Thyroid 19(11), 1159-1165 (2009). doi:10.1089/thy.2009.0274

11. Brenta, G., Schnitman, M., Bonnahon, L., Besuschio, S., Zuk, C., De Barrio, G., Peruzzotti, C., Saubidet, G.: Evaluation of innovative skin-marking technique performed before thyroid ultrasound-guided fine-needle aspiration biopsies. Endocr Pract 8(1), 5-9 (2002). doi:EP01001.OR 
12. DeLong, E.R., DeLong, D.M., Clarke-Pearson, D.L.: Comparing the areas under two or more correlated receiver operating characteristic curves: a nonparametric approach. Biometrics 44(3), 837-845 (1988).

13. Robin, X., Turck, N., Hainard, A., Tiberti, N., Lisacek, F., Sanchez, J.C., Muller, M.: pROC: an opensource package for $\mathrm{R}$ and $\mathrm{S}+$ to analyze and compare ROC curves. BMC Bioinformatics 12, 77 (2011). doi:10.1186/1471-2105-12-77

14. Wang, Z., Vyas, C.M., Van Benschoten, O., Nehs, M.A., Moore, F.D., Jr., Marqusee, E., Krane, J.F., Kim, M.I., Heller, H.T., Gawande, A.A., Frates, M.C., Doubilet, P.M., Doherty, G.M., Cho, N.L., Cibas, E.S., Benson, C.B., Barletta, J.A., Zavacki, A.M., Larsen, P.R., Alexander, E.K., Angell, T.E.: Quantitative Analysis of the Benefits and Risk of Thyroid Nodule Evaluation in Patients >/=70 Years Old. Thyroid 28(4), 465-471 (2018). doi:10.1089/thy.2017.0655

15. Yoon, J.H., Lee, H.S., Kim, E.K., Moon, H.J., Kwak, J.Y.: Malignancy Risk Stratification of Thyroid Nodules: Comparison between the Thyroid Imaging Reporting and Data System and the 2014 American Thyroid Association Management Guidelines. Radiology 278(3), 917-924 (2016). doi:10.1148/radiol.2015150056

16. Persichetti, A., Di Stasio, E., Guglielmi, R., Bizzarri, G., Taccogna, S., Misischi, I., Graziano, F., Petrucci, L., Bianchini, A., Papini, E.: Predictive Value of Malignancy of Thyroid Nodule Ultrasound Classification Systems: A Prospective Study. J Clin Endocrinol Metab 103(4), 1359-1368 (2018). doi:10.1210/jc.2017-01708

17. Lauria Pantano, A., Maddaloni, E., Briganti, S.I., Beretta Anguissola, G., Perrella, E., Taffon, C., Palermo, A., Pozzilli, P., Manfrini, S., Crescenzi, A.: Differences between ATA, AACE/ACE/AME and ACR TI-RADS ultrasound classifications performance in identifying cytological high-risk thyroid nodules. Eur J Endocrinol 178(6), 595-603 (2018). doi:10.1530/EJE-18-0083

18. Vera, M.I., Merono, T., Urrutia, M.A., Parisi, C., Morosan, Y., Rosmarin, M., Schnitman, M., Brites, F., Grisendi, S., Serrano, M.S., Luciani, W., Serrano, L., Zuk, C., De Barrio, G., Cejas, C., Faingold, M.C., Brenta, G.: Differential profile of ultrasound findings associated with malignancy in mixed and solid thyroid nodules in an elderly female population. J Thyroid Res 2014, 761653 (2014). doi:10.1155/2014/761653

19. Shi, C., Li, S., Shi, T., Liu, B., Ding, C., Qin, H.: Correlation between thyroid nodule calcification morphology on ultrasound and thyroid carcinoma. J Int Med Res 40(1), 350-357 (2012). doi:10.1177/147323001204000136

20. Wang, Z., Zhang, H., Zhang, P., He, L., Dong, W.: Diagnostic value of ultrasound-detected calcification in thyroid nodules. Ann Acad Med Singapore 43(2), 102-106 (2014).

21. Grani, G., Lamartina, L., Ascoli, V., Bosco, D., Biffoni, M., Giacomelli, L., Maranghi, M., Falcone, R., Ramundo, V., Cantisani, V., Filetti, S., Durante, C.: Reducing the Number of Unnecessary Thyroid Biopsies While Improving Diagnostic Accuracy: Toward the "Right" TIRADS. J Clin Endocrinol Metab 104(1), 95-102 (2019). doi:10.1210/jc.2018-01674

22. Xu, T., Wu, Y., Wu, R.X., Zhang, Y.Z., Gu, J.Y., Ye, X.H., Tang, W., Xu, S.H., Liu, C., Wu, X.H.: Validation and comparison of three newly-released Thyroid Imaging Reporting and Data Systems for cancer risk determination. Endocrine 64(2), 299-307 (2019). doi:10.1007/s12020-018-1817-8

23. Tappouni, R.R., Itri, J.N., McQueen, T.S., Lalwani, N., Ou, J.J.: ACR TI-RADS: Pitfalls, Solutions, and Future Directions. Radiographics 39(7), 2040-2052 (2019). doi:10.1148/rg.2019190026 


\section{FIGURE LEGENDS}

Figure 1: Selection of the studied population 
Table 1: US characteristics and cytological results of the total group of nodules $(n=1867)$

\begin{tabular}{|l|c|}
\hline US characteristics & $\mathrm{N}(\%)$ \\
\hline Solid nodules & $1400(75 \%)$ \\
\hline $\begin{array}{l}\text { Echogenicity (mildly } \\
\text { hypoechoic) }\end{array}$ & $702(37.5 \%)$ \\
\hline $\begin{array}{l}\text { Echogenicity (markedly } \\
\text { hypoechoic) }\end{array}$ & $68(3.8 \%)$ \\
\hline Presence of halo & $542(29 \%)$ \\
\hline Microcalcifications & $252(13.5 \%)$ \\
\hline Macrocalcifications & $285(15 \%)$ \\
\hline $\begin{array}{l}\text { Irregular margins } \\
\text { III }\end{array}$ & $368(20 \%)$ \\
\hline Taller than wide & $134(7.2 \%)$ \\
\hline $\begin{array}{l}\text { Predominantly-central } \\
\text { vascularization pattern }\end{array}$ & $286(15 \%)$ \\
\hline $\begin{array}{l}\text { Bethesda System Classification } \\
\text { VI }\end{array}$ & $26(1.4 \%)$ \\
\hline
\end{tabular}


Table 2: Comparison of malignant cytology within the categories of each US classification

\begin{tabular}{|c|c|c|c|}
\hline $\begin{array}{c}\text { ACR TI-RADS } \\
(n=1587)\end{array}$ & $\mathrm{n}(\%)$ & $\begin{array}{c}\text { Malignant cytology } \\
\mathrm{n}(\%)\end{array}$ & $p$ for trend \\
\hline TR1 & $39(2.4 \%)$ & - & \\
\hline TR2 & $105(6.6 \%)$ & $2(1.9 \%)$ & \\
\hline TR3 & $422(26.6 \%)$ & $8(1.9 \%)$ & \\
\hline TR4 & $730(46.0 \%)$ & $23(3.1 \%)$ & \\
\hline TR5 & $291(18.3 \%)$ & $17(5.8 \%)$ & 0.028 \\
\hline \multicolumn{4}{|l|}{ ATA $(n=1285)$} \\
\hline Benign & $13(1.0 \%)$ & - & \\
\hline Very low suspicion & $20(1.6 \%)$ & $1(5 \%)$ & \\
\hline Low suspicion & $716(55.7 \%)$ & $9(1.3 \%)$ & \\
\hline Intermediate suspicion & $281(21.9 \%)$ & $9(3.2 \%)$ & \\
\hline High suspicion & $255(19.8 \%)$ & $17(6.7 \%)$ & $<0.001$ \\
\hline \multicolumn{4}{|l|}{ AACE/ACE/AME $(n=1495)$} \\
\hline Class 1 & $31(2.1 \%)$ & $2(6.5 \%)$ & \\
\hline Class 2 & $725(48.5 \%)$ & $11(1.5 \%)$ & \\
\hline Class 3 & $739(49.4 \%)$ & $35(4.7 \%)$ & $<0.001$ \\
\hline
\end{tabular}


Table 3. Paired-comparison of ROC curves

\begin{tabular}{|c|c|c|}
\hline$(\mathrm{AUC})$ & $\mathrm{Z}$ value & $p$ value \\
\hline \multicolumn{3}{|c|}{ Unclassifiable malignant nodules categorized in the lowest risk category $(1234, M=50)$} \\
\hline $\begin{array}{l}\text { ATA }(0.49) \text { vs ACR TI-RADS } \\
(0.62)\end{array}$ & 2.65 & 0.008 \\
\hline ATA vs AACE/ACE/AME (0.59) & 2.28 & 0.022 \\
\hline $\begin{array}{l}\text { AACE/ACE/AME vs ACR TI- } \\
\text { RADS }\end{array}$ & -0.58 & 0.56 \\
\hline \multicolumn{3}{|c|}{ Excluding all unclassifiable nodules $(n=1218, M=34)$} \\
\hline $\begin{array}{l}\text { ATA }(0.68) \text { vs ACR TI-RADS } \\
(0.61)\end{array}$ & -2.56 & 0.01 \\
\hline ATA vs AACE/ACE/AME (0.59) & -2.88 & $<0.001$ \\
\hline $\begin{array}{l}\text { AACE/ACE/AME vs ACR TI- } \\
\text { RADS }\end{array}$ & -0.73 & 0.46 \\
\hline
\end{tabular}

AUC, area under the curve.

Supplementary Table 1. Cut-off values in each US system as predictor of malignancy.

\begin{tabular}{|c|c|c|c|c|c|c|c|c|}
\hline Score & $\begin{array}{c}\text { Cut-off } \\
\text { level }\end{array}$ & AUC & S & SP & PPV & NPV & $\begin{array}{c}\text { OR } \\
(95 \% \text { CI })\end{array}$ & LR \\
\hline $\begin{array}{c}\text { ACR TI-RADS } \\
(\mathrm{n}=1587, \\
\text { malignant=50) }\end{array}$ & TR5 & $\begin{array}{c}0.61 \\
(0.52-0.71)\end{array}$ & $34 \%$ & $82 \%$ & $6 \%$ & $97 \%$ & $2.36(1.3-4.3)$ & 1.90 \\
\hline $\begin{array}{c}\text { ATA } \\
(\mathrm{n}=1285, \\
\text { malignant=36) }\end{array}$ & $\begin{array}{c}\text { High } \\
\text { suspicion }\end{array}$ & $\begin{array}{c}0.68 \\
(0.58-0.77)\end{array}$ & $47 \%$ & $80 \%$ & $6.6 \%$ & $98 \%$ & $3.8(1.9-7.4)$ & 2.47 \\
\hline $\begin{array}{c}\text { AACE/ACE/AME } \\
(\mathrm{n}=1495, \\
\text { malignant=48) }\end{array}$ & Class 3 & $\begin{array}{c}0.59 \\
(0.50-0.68)\end{array}$ & $73 \%$ & $51 \%$ & $4.7 \%$ & $98 \%$ & $2.84(1.4-5.4)$ & 1.49 \\
\hline
\end{tabular}

AUC $=$ Area under the curve. $S=$ Sensitivity. $S P=$ =Specificity. $\mathbf{P P V}=$ positive predictive value.

$N P V=$ Negative predictive value. $O R=$ odds ratio. $L R=$ likelihood ratio. 
Figure 1: Selection of the studied population

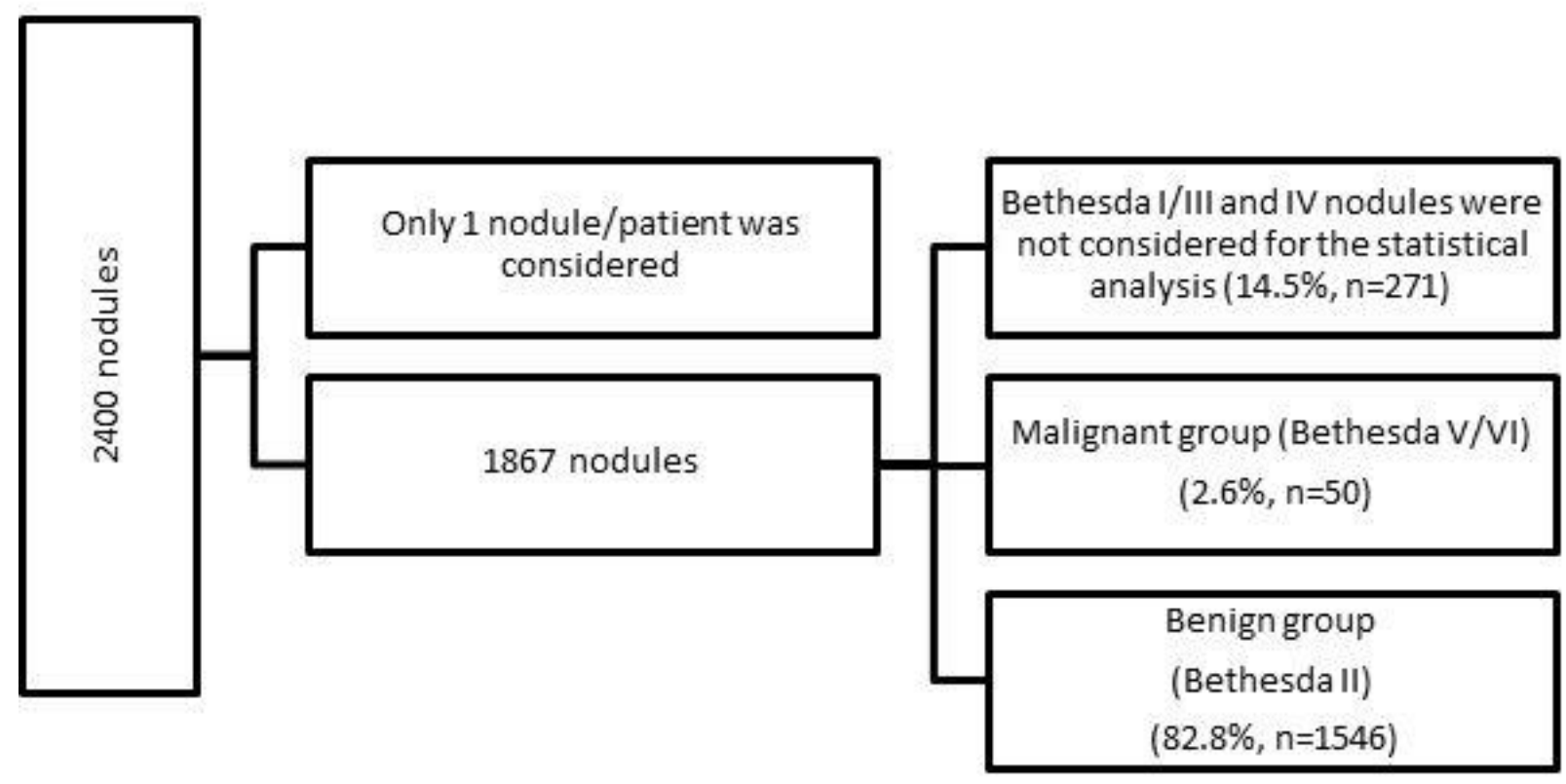

